\title{
THE THIRD AXIOM OF COUNTABILITY FOR ABELIAN GROUPS
}

\author{
PAUL HILL ${ }^{1}$
}

\begin{abstract}
Three different definitions of the third axiom of countability for abelian $p$-groups are shown to be equivalent. The main interest in this stems from the fact that the third axiom of countability characterizes one of the most important classes of abelian groups. Moreover, the equivalence of two of these definitions validates the proof of Theorem 67 in P. Griffith's Infinite abelian group theory.

As a further application of our method of proof, we show that every torsionfree abelian group satisfies the third axiom of countability with respect to purity.
\end{abstract}

I. Following the established terminology of [3] and [4], we say that a subgroup $H$ of an abelian $p$-group is a nice subgroup of $G$ if $p^{\alpha}(G / H)=\left\langle p^{\alpha} G, H\right\rangle / H$ for each ordinal $\alpha$. In accordance with [4], as well as [5] and [6], $G$ is said to satisfy the third axiom of countability if there exists a collection $\mathcal{C}$ of nice subgroups of $G$ such that

(0) $0 \in \mathcal{C}$.

(1) $\mathcal{C}$ is closed with respect to the group union of an arbitrary number of groups.

(2) If $A \in \mathcal{C}$ and $K$ is any countable subgroup of $G$, there exists $B \in \mathcal{C}$ such that $B / A$ is countable and $B \supseteq\langle A, K\rangle$.

It was first shown in [4] that the reduced abelian p-groups satisfying the preceding third axiom of countability are uniquely determined by their Ulm invariants and that these groups coincide precisely with the totally projective $p$-groups introduced by $R$. Nunke in [7]. Also, a short proof was given by the author in [5] that the $T$-groups studied by $P$. Crawley and A. Hales [1] satisfy the third axiom of countability.

In his monograph, Griffith [3] chose a slightly different definition for Axiom 3. He replaced condition (1) of the axiom by the following weaker condition.

$\left(1^{\prime}\right) \mathcal{C}$ is closed with respect to ascending unions.

In the development of the properties and structure of Axiom 3 groups, the most noticeable difference between these two definitions occurs in the proof that a direct summand of an Axiom 3 group is again an Axiom 3 group. The direct summand result is Theorem 67 in [3]. It is well known that the proof of Theorem 67 is erroneous because Griffith's axiom cited above does not imply that $\mathcal{C}_{\boldsymbol{H}}$ is closed with respect to ascending unions as claimed in [3] although the stronger axiom formulated by Hill would. For a more detailed discussion of this matter, we refer to

Received by the editors January 16, 1975 and, in revised form, December 26, 1979.

AMS (MOS) subject classifications (1970). Primary 20K10, 20K20; Secondary 20E15.

Key words and phrases. Third axiom of countability, totally projective group, ascending chain of nice subgroups, Ulm invariant, torsionfree group, pure subgroup.

${ }^{1}$ This research was supported by National Science Foundation grant GP-38071X. 
the remarks of E. Walker in [8]. We shall give here a simple direct proof that the two definitions are equivalent. The equivalence was actually established in [4] and [2], but the proof of the equivalence heretofore has required first the development of further significant results such as Ulm's theorem for third countable groups. In fact, L. Fuchs suggested in [2, p. 84] that a direct proof would not be easy, and E. Walker expressed doubt about the existence of a direct proof in [8].

Our proof is designed so that we can include with no additional effort another equivalent definition of the third axiom of countability due to Fuchs. A p-primary group $G$ is said to satisfy condition (F) if there exists an ascending chain $0=G_{0} \subseteq$ $G_{1} \subseteq \cdots \subseteq G_{\alpha} \subseteq \cdots \subseteq G_{\gamma}=G$ of nice subgroups $G_{\alpha}$ such that

(a) $\left|G_{\alpha+1} / G_{\alpha}\right|=p$ for each $\alpha$, and

(b) $G_{\beta}=\cup_{\alpha<\beta} G_{\alpha}$ whenever $\beta<\gamma$ is a limit ordinal.

For convenience, we let $(\mathrm{H})$ and $(\mathrm{G})$ denote, respectively, Hill's and Griffith's criterion for third countable groups.

THeOREM 1. The conditions (H), (G), and (F) are equivalent for all p-primary abelian groups.

Proof. The implication $(\mathrm{H}) \Rightarrow(\mathrm{G})$ is trivial and the implication $(\mathrm{G}) \Rightarrow(\mathrm{F})$ is rather immediate because criterion (G) obviously implies the existence of an ascending chain $0=G_{0} \subseteq G_{1} \subseteq \cdots \subseteq G_{\alpha} \subseteq \cdots \subseteq G_{\gamma}=G$ of nice subgroups $G_{\alpha}$ such that (b) is satisfied and $\left|G_{\alpha+1} / G_{\alpha}\right|<\aleph_{0}$. Condition (a) can be obtained by an appropriate refinement of the given chain. In this connection, observe that a finite extension of a nice subgroup is always again a nice subgroup. Thus we need only show that $(\mathrm{H})$ follows directly from (F). Hence we shall assume (F). For each $\alpha<\gamma$, choose $x_{\alpha} \in G_{\alpha+1}$ to be proper with respect to $G_{\alpha}$. In other words, $x_{\alpha}$ is chosen so that it has maximal height in $x_{\alpha}+G_{\alpha}$ since $G_{\alpha}$ is a nice subgroup of $G$. Clearly, $G=\left\langle x_{\alpha}\right\rangle_{\alpha<\gamma}$ in view of conditions (a) and (b) on the chain of subgroups $G_{\alpha}$. Furthermore, if $g$ is a nonzero element of $G$, then $g$ can be represented uniquely in the form

$$
g=t_{1} x_{\alpha(1)}+t_{2} x_{\alpha(2)}+\cdots+t_{k} x_{\alpha(k)},
$$

where $0<t_{i}<p$, for each $i$, and $\alpha(1)<\alpha(2)<\cdots<\alpha(k)$.

Our purpose is to show that $G$ has a collection $\mathcal{C}$ of nice subgroups that satisfies the conditions of $(\mathrm{H})$, and we shall do this in terms of the selected generators $x_{\alpha}$. For notational convenience, let $T$ denote the set of ordinals less than $\gamma$ (which was used to index the chain of subgroups $G_{\alpha}$ and the generators $x_{\alpha}$ of $G$ ). Call a subset $S$ of $T$ a closed subset if whenever $\alpha \in S$ and $g=p x_{\alpha}$ is not zero, then $\alpha(i)$, too, belongs to $S$ for each $i$ employed in the representation (*) of the element $g$. Equivalently, the subset $S$ of $T$ is closed if each nonzero element of the subgroup $H=\left\langle x_{\alpha}\right\rangle_{\alpha \in S}$ of $G$ has the representation (*) based on the exhibited generators of $H$. It is immediate (from the initial description of closure) that the union of an arbitrary number of closed subsets of $T$ is again closed. Moreover, it is easy to see that any countable subset of $T$ has a countable closure in $T$. We merely adjoin the required new generators, and then repeat the process a countable number of times. It remains only to show that $H=\left\langle x_{\alpha}\right\rangle_{\alpha \in S}$ is a nice subgroup of $G$ whenever $S$ is a 
closed subset of $T$ because the collection $\mathcal{C}$ of all such subgroups certainly satisfies the conditions of Hill's criterion $(\mathrm{H})$.

Suppose that $S$ is a closed subset of $T$. We want to show that $H=\left\langle x_{\alpha}\right\rangle_{\alpha \in S}$ is a nice subgroup of $G$. We may, of course, assume that $H \neq G$. Choose an arbitrary nonzero coset $x+H$ and select a representative $g$ from this coset whose representation (*) does not involve elements of $H$. We claim that $g$ has maximal height in $G$ among the elements of the coset $g+H$. This follows immediately from the observation that the height of the element $g$ with representation (*) is the minimum of the heights of the elements $x_{\alpha(i)}$ in (*). This observation is readily established by induction on $k$. This completes the proof of the theorem since a subgroup is nice if each coset contains an element with maximal height.

REMARK. In the proof of Theorem 67 in [3] one can now assume by virtue of Theorem 1 that $e_{H}$ satisfies criterion $(H)$, as well as $(G)$, and the proof then survives.

II. Using the proof of Theorem 1 as a model, we demonstrate in this section that every torsionfree group $G$ satisfies the third axiom of countability with respect to pure subgroups. Here, the third axiom means the original third axiom $(\mathrm{H})$ except that pure subgroups replace nice subgroups. There exists a collection $\mathcal{C}$ of pure subgroups satisfying conditions (0), (1), and (2).

THEOREM 2. Every torsionfree abelian group is third countable with respect to pure subgroups.

Proof. Since the result is trivial for countable groups, let $G$ denote an uncountable torsionfree group. With purity being an inductive property, there exists an ascending chain, indexed by an uncountable limit ordinal $\gamma$,

$$
0=G_{0} \subseteq G_{1} \subseteq \cdots \subseteq G_{\alpha} \subseteq \cdots \subseteq G_{\gamma}=G
$$

of pure subgroups of $G$ such that

(a') $G_{\alpha+1} / G_{\alpha} \subseteq Q$ (has rank 1) for each $\alpha$ and

(b') $G_{\beta}=\cup_{\alpha<\beta} G_{\alpha}$ whenever $\beta<\gamma$ is a limit ordinal.

For each ordinal $\alpha<\gamma$, choose a representative $x_{\alpha}$ from the set $G_{\alpha+1} \backslash G_{\alpha}$. Since $G$ is torsionfree, each subset of $G$ is contained in a unique minimal pure subgroup. Denote by $\mathcal{P}(S)$ the pure subgroup generated by $S$. It is a simple exercise to verify by induction on $\beta$ for each $\beta \leqslant \gamma$ that $G_{\beta}=\mathscr{P}\left(x_{\alpha}: \alpha<\beta\right)$. As before, let $T$ denote the set of ordinals less than $\gamma$. To proceed, we need to select some distinguished subsets of $T$ having some kind of closure property. The notion of closure here, of course, needs to be different from that used in Theorem 1 since the pure subgroups are now the distinguished subgroups of $G$.

A summation $\sum_{i=0}^{r} n_{i} x_{\alpha(i)}$ with integral coefficients $n_{i}$ is said to be descending if $\alpha(0)>\alpha(1)>\cdots>\alpha(r)$. Call a subset $S$ of $T$ closed if: the existence of a descending summation $\sum_{i=0}^{r} n_{i} x_{\alpha(i)}$ with the sum in $n G$ and $\alpha(0) \in S$ for a positive integer $n$ implies the existence of a descending summation $\Sigma_{i=0}^{t} m_{i} x_{\beta(i)}$ with sum in $n G$ satisfying the conditions (i) $\alpha(0)=\beta(0)$, (ii) $n_{0}=m_{0}$, and (iii) $\beta(i) \in S$ for each $i<t$. Notice that for an arbitrary subset $S$ of $T$ the closure property relative to all summations $\sum_{i=0}^{r} n_{i} x_{\alpha(i)}$ with sum in $n G$ and $\alpha(0), n(0)$ and $n$ fixed can be satisfied 
by adjoining only a finite number of elements to $S$, namely, $\alpha(1), \alpha(2), \ldots, \alpha(r)$ for a particular summation if they do not already belong to $S$. Since there are only a countable number of triples $(\alpha(0), n(0), n)$ when $\alpha(0)$ has a countable range, it follows that any countable subset of $T$ can be expanded, by repeating the annexation of a finite number of elements a countable number of times, to a countable closed subset of $T$.

We claim that the following collection of pure subgroups satisfies conditions ( 0 ), (1) and (2) of axiom (H), and therefore $G$ satisfies the third axiom of countability with respect to pure subgroups.

$$
\mathcal{C}=\{\mathscr{P}(S): S \text { is a closed subset of } T\} .
$$

Condition (0) is satisfied by the convention $\mathscr{P}(\varnothing)=0$. The main step in proving that conditions (1) and (2) hold is the observation that $\mathscr{P}\left(S_{1} \cup S_{2}\right)=$ $\left\langle\mathcal{P}\left(S_{1}\right), \mathcal{P}\left(S_{2}\right)\right\rangle$ whenever $S_{1}$ and $S_{2}$ are closed subsets of $T$. This is equivalent to recognizing that $\left\langle\mathcal{P}\left(S_{1}\right), \mathcal{P}\left(S_{2}\right)\right\rangle$ is a pure subgroup whenever $S_{1}$ and $S_{2}$ are closed. To prove purity, suppose that $n g \in\left\langle\mathscr{P}\left(S_{1}\right), \mathscr{P}\left(S_{2}\right)\right\rangle$ where $g \in G$, and let $n g=a+b$ where $a \in \mathcal{P}\left(S_{1}\right)$ and $b \in \mathcal{P}\left(S_{2}\right)$. For a suitable positive integer $m$, we have $m n g=m a+m b=\sum_{i=0}^{r} n_{i} x_{\alpha(i)}$ where the summation descends and, for each $i, \alpha(i)$ is either in $S_{1}$ or $S_{2}$. Without loss of generality, we may assume that $\alpha(0) \in S_{1}$. Now, since $S_{1}$ is closed, there exists $x \in \mathscr{P}\left(S_{1}\right)$ such that $m n(g-x)=$ $\sum_{i=0}^{t} m_{i} x_{\beta(i)}$ is a descending summation with $\beta(i) \in S_{1} \cup S_{2}$ for each $i$ and $\beta(0)<\alpha(0)$. By transfinite induction, we may assume that $g-x \in$ $\left\langle\mathscr{P}\left(S_{1}\right), \mathscr{P}\left(S_{2}\right)\right\rangle$ and therefore $g \in\left\langle\mathscr{P}\left(S_{1}\right), \mathscr{P}\left(S_{2}\right)\right\rangle$.

Since the union of any number of closed subsets is again closed (in view of our definition of closure) and since purity is an inductive property, the equation $\mathscr{P}\left(S_{1} \cup S_{2}\right)=\left\langle\mathscr{P}\left(S_{1}\right), \mathscr{P}\left(S_{2}\right)\right\rangle$ yields condition (1) of axiom (H) because $\left\langle\mathscr{P}\left(S_{i}\right)\right\rangle_{i \in I}=\mathscr{P}\left(\bigcup_{i \in I} S_{i}\right)$.

It remains only to prove condition (2). And it suffices to prove that each element $g \in G$ belongs to $\mathscr{P}(S)$ for some countable closed set $S$ because $\mathscr{P}(S)$ is countable if $S$ is. However, for some positive integer $n$, we can write $n g=$ $\sum_{i=0}^{r} n_{i} x_{\alpha(i)}$. There is a countable closed set $S$ containing $\alpha(i)$ for each $i$ since any finite set is contained in a countable closed set. Obviously, $g \in \mathcal{P}(S)$, and the proof of the theorem is complete.

\section{REFERENCES}

1. P. Crawley and A. Hales, The structure of torsion abelian groups given by presentations, Bull. Amer. Math. Soc. 74 (1968), 954-956.

2. L. Fuchs, Infinite abelian groups, vol. II, Academic Press, New York, 1973.

3. P. Griffith, Infinite abelian group theory, Univ. of Chicago Press, Chicago and London, 1970.

4. P. Hill, On the classification of abelian groups, xeroxed manuscript, 1967.

5. $\longrightarrow$ A countability condition for pimary groups presented by relations of length two, Bull. Amer. Math. Soc. 75 (1969), 780-782.

6. $\_$On the decomposition of certain infinite nilpotent groups, Math. Z. 113 (1970), 237-248.

7. R. J. Nunke, Homology and direct sums of countable abelian groups, Math. Z. 101 (1967), 182-212.

8. E. Walker, Ulm's theorem for totally projective groups, Proc. Amer. Math. Soc. 37 (1973), 387-392.

Department of Mathematics, Florida State University, Tallahassee, Florida 32306

Current address: Department of Mathematics, Auburn University, Auburn, Alabama 36830 